\title{
A Descriptive Study on Male Breast Cancer and Its Contingency in Pakistan
}

\author{
Aimen Khan and Sadia Zia* \\ Department of Biotechnology, University of Central Punjab, Lahore, Pakistan \\ *Corresponding author: Sadia Zia, Department of Biotechnology, University of Central Punjab, Lahore, Pakistan
}

\section{ARTICLE INFO}

Received: 㓞 September 26, 2019

Published:

Citation: Aimen Khan, Sadia Zia. A Descriptive Study on Male Breast Cancer and Its Contingency in Pakistan. Biomed J Sci \& Tech Res 21(4)-2019. BJSTR. MS.ID.003645.

Keyword: Pakistan; Male Breast Cancer; Treatment Strategies

\begin{abstract}
Male breast cancer is one of the rarer diseases, which accounts for less than $1 \%$ of cancer registries worldwide. Male breast cancer is mostly caused by genetic mutations in BRCA1 and BRCA2. Several other -non-genetic factors contribute to the onset of MBC. The methods opted for diagnosis and screening MBC are quite same as for FBC. Its percentage is increasing day by day. In Pakistan the prevalence of MBC is regarded as approximately 2 males per 100 females. MBC is mostly pronounced in the seventh decade state. The symptoms of MBC mostly imitate the symptoms of breast cancer of post-menopausal women. Treatment strategies employed for the management of MBC are same as for FBC. But males show deviation in prognosis of the disease as compared to females. The overall survival rate for $\mathrm{MBC}$ is about 5-8 years. $\mathrm{MBC}$ is affected by Racial distribution and ethnicity. NGS and novel identification techniques are devised to discuss MBC at early stage which is otherwise identified at late stages. Modern therapeutic techniques are devised to come over the limitations of the conventional treatment methods. Overall this review provides elaborate information about the risk factors, epidemiology, prognostic factors, stages and diagnosis along with treatment and future perspective of MBC.
\end{abstract}

\section{Introduction}

Cancer is one of the most researched topics of the age. Since last twenty years, a number of considerable advancements have been done in discernment of breast cancer so that it can be pronounced at initial stages which will ultimately lead to better management [1]. According to GLOBCAN cancer stats of 2018, breast cancer is the second most rampant cancer and is one of the top 10 most prevalent cancers [2].

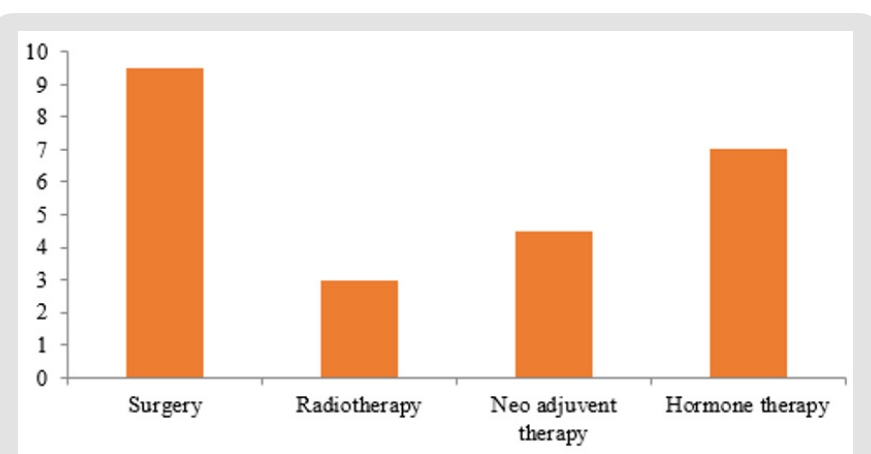

Figure 1: Treatment employment stats (Approx).
Breast cancer in males is a scanty as compared to females [3]. Out of all the breast cancer registrations, registration for male breast cancer ranges from $0.6 \%-01 \%$. The reason for these minimal numbers of registrations is lack of awareness and abstruse concept of MBC in the society. The breast tissue mass is comparatively low in males as compared to females and distinction in hormonal medium leads to low pervasiveness of MBC [4]. The SEER (Surveillance, Epidemiology, and End Results) reported during 1973-2000 that breast cancer was most prevalent in the age group 52-71 (Figure 1).

Occurrence of breast cancer in both male and female have increased by $20 \%$ since the last 2 decades. The global occurrence of male breast cancer has been approximately increased from 1 to 1.5 per 100,000 men. The rate of male breast cancer has become 1 in 1000 males in USA [5]. This pervasiveness is much higher in central African and Asian countries [6]. In Pakistan the frequency of $\mathrm{MBC}$ is recorded as 2 men per every 100 females [7]. The frequency of MBC depends widely upon the geographical distribution and ethnicity of individuals [8,9]. Approximately, 2500 cases of breast cancer are reported in men in the year 2017 [10]. 
Individuals with a positive family history are more likely to develop MBC or non-breast tumors by 15-20\% [11]. Gynecomastia (enlargement of male breast should not be confused with MBC, both of the diseases are based on distinct concepts whereas it may be found in patients with MBC $[12,13]$. Genetics play an important role in MBC susceptibility [14]. Genetic mutations in BRCA1 and BRCA2 increase the likelihood of MBC [15]. BRCA2 mutations are more frequently detected [16].

Several other factors that facilitate the onset of MBC are klinefelter syndrome, hepatic manifestations and diseases of male genitals i.e. prostate cancer, cryptorchidism etc, these disorders are found complementary to male breast cancer $[17,18]$. Symptoms and signs of MBC are seen in juvenile ages in individuals with klinefelter syndrome. Klinefelter syndrome increases the probability of male breast cancer by $50 \%$ [19].

CHEK2 and PALB2 germline mutation also increase the plausibility of MBC $[20,21]$. The identification of MBC is done by either mammography or ultrasound, therefore the clinicalpathological layout of MBC diverges with the female layout [22,23] but biopsy, genetic testing, histological analysis and biomarker identification are more explicit and reliable methods for the detection of MBC.

The characteristics of MBC mimic the characteristics of postmenopausal females [24,25]. MBC is substantially hormone (estrogen) receptor positive and her-2/neu negative, MBC predominantly consist of ductal carcinomas and rarely develop lobular carcinoma whereas lobular carcinomas are more widespread in females with breast cancer [26] in MBC BRCA2 mutations are often overly expressed in contrast to BRCA1 mutations [27].

The treatment progression of MBC is same as for the female breast cancer. Surgical procedures are prioritized in the amelioration of MBC which is further accompanied with systemic chemotherapies, radiotherapies, and hormonal therapies. Individuals with positive hormone (estrogen, progesterone) receptor tumors are treated with hormonal therapies, these therapies work by enhancing the expression of [Her-2/neu], such patients also undergo tamoxifen and aromatase hormonal therapies. After surgical excisions adjuvant therapies are recommended depending upon the medical condition of the patients [28].

The survival rates have drastically increased in the last couple of decades [29] with the latest advancements in the field of genetic testing. Multiple genes that are associated with cancer are identified and discovered by using NGS (next generation sequencing) at fairly low cost, these genes (CDH1, PTEN, STK11, TP53 and mismatch genes hMLH1, hMLH2) are available for genetic testing (93).

\section{Symptoms and Signs}

The symptoms and signs of the male breast cancer appear at a much-delayed stage. As considering the anatomy of male breast cancer it should be easily diagnosed but it's not. The reason is that MBC becomes symptomatic at advanced stages and also not enough information or research is available (73). Painful or painless lumps, Dimpling breast ,Puckering over skin of the breast, Change in size, Rash or redness on the area around breast ,Nipple discharge ,Redness over nipples or bleeding ,Nipple retraction; seeing any of these potential features and moreover if these symptoms persist one should immediately consult a doctor without a further delayed.

\section{Staging of Male Breast Cancer}

The staging of the breast cancer in male is no more different than female, the method adopted for evaluation are the same. Size of the tumor, Metastasis and Nodal involvement These features are also abbreviated as (TNM). The most important ones are the tumor size and nodal involvement, on the basis of their values the stage of cancer is majorly determined [30].

\section{Diagnosis and Screening}

The steps followed by the diagnosis of MBC are listed below, starting from the primary to advanced diagnostic techniques, Personal examination, and Clinical examination and Advanced screening techniques. To clear out any confusions a differential diagnosis is run, for differentiating male breast cancer from Fibromatosis, Gynecomastia, Psuedogynecomastia, Infections, Lymphoma, Other metastatic disease.

MRI: MRI abbreviated as magnetic resonance imaging is also used to obtain detailed tissue images of both the breasts which is vital to determine the extent of disease.

Mammography \& Ultrasonography: When it comes to diagnosis of male breast cancer, about $90-95 \%$ of the cases of male breast cancer are reported by identification through mammography [75] Ultrasonography is carried out if there is a sign of nodal involvement. Both these techniques are employed if the physical examinations fail to detect any tumors in the breast [76].

Biopsy: There are several types of biopsies depending upon the position of the tumor and its size ,Incisional biopsy (removal of a part of lump),Excisional biopsy (removal of an entire lump),FNA biopsy (fine needle aspiration biopsy) ,Core biopsy (removal of tissues with a wide needle).in case of dealing with definitive histological analysis core biopsy is preferred.

Hormone Receptor Tests: Further testing is done to check either the carcinoma occurring is positive or negative for the hormone receptors.

Estrogen/Progesterone Test: A test is run to evaluate the amount of hormones estrogen and progesterone in the cancer tissues. If high amounts of estrogen and progesterone are found in the tissues, then it is labeled as estrogen positive or progesterone positive depending upon the levels of hormones identified. Most the MBC patients are mostly estrogen positive, because out of all the risk factors disturbed estrogen hormone levels show greater prevalence.

Her-2 Test: It is a test that is used to check the amount of her$2 /$ neu proteins or genes in the cancer tissues. 


\section{Her-2 enriched}

This type of cancer shows high levels of her-2/neu protein and is mostly found in women and is very rare in males.

\section{Advanced Methods in Staging}

Once the presence of cancer is confirmed furthermore tests such as chest scans and bone scans are run to check the extent of the tumor and for better staging of the disease.

\section{Treatment of Male Breast Cancer}

Surgical incision is the main first line treatment for the male breast cancer the approach is similar in both the genders.

Mastectomy: Mastectomy is commonly defined as the removal of breast. This is the most prioritized treatment in males as mastectomy is not linked to aesthetic reasons.

A. Radiotherapy: These radiotherapies are prescribed in some special cases such as when there are less than four positive axillary lymph nodes; Tumor size is smaller than 5 or up to three positive nodes with extra capsular adjunct.

B. Chemotherapy: chemotherapies have proven to be beneficial for the prognosis of MBC. Antracycline-based and anthracycline paired with texans are given to patients without and with lymph node involvement respectively (83). Trastuzumab is commonly regarded as Herceptin, is a monoclonal antibody often prescribed for the treatment of male breast cancer.

C. Endocrine therapy: Tamoxifien is the most widely used drug as an adjuvant therapy, it is usually prescribed as $20 \mathrm{~g}$ dosage for approx. 5 years, this medication is specifically preferred for estrogen positive patients. Adjuvant endocrine therapy is prescribed alone or in combination with chemotherapy for male breast cancer that is pronounced at initial stages (84). Recent studies conducted on adjuvant endocrine therapy suggest that it should be the primary or first line treatment for male breast cancer as most of the cases are abundant in hormone receptors. Other adjuvant therapies are also recommended in positive auxiliary lymph node or substantial tumors.AI therapy (Aromatase inhibitor therapy) is also employed in adjuvant endocrine therapy but its scope is limited therefore Tamoxifen therapy is preferred over AI therapy [31,32].

Advanced and Metastatic Disease: Out of all the male breast cancer cases approximately $5 \%$ to $15 \%$ are identified as metastatic in nature. According to the cases reported the most prevalent location of metastasis is around the lungs and liver, this type of metastasis accounts for approximately $75 \%$ of all the cases. The other most extensive localities for metastasis are bones and skin and they account for about 20 and $4 \%$ of all the cases respectively (85).

\section{Prognostic Factors}

Although these are the available treatments, but every patient behaves differently to these therapies this is because of the variable prognostic factors. Age hinders the prognosis in male breast cancer, as it is diagnosed in late ages therefore the expected results of the therapies are not often obtained. Stage of the cancer plays a key role in prognosis but male breast cancer is mostly pronounced at late stages which limits the number of treatments employed .Estrogen/ progestron receptor levels in the tissue, males respond better to hormone therapies than females, hence they show better prognosis when it comes to estrogen positive cases .Type of the cancer diagnosed widely affects the prognosis luminal A type shows better prognosis then luminal $B$ and triple negetive in males is a very difficult type to cure in males .Overall health plays a key role not only in prognosis but in the recurrence of the disease.

\section{Novel Identification Techniques}

MicroRNA expression profiling, (PET-CT) Scan and NGS (next generation sequencing) ae some of the nova identification techniques but these are only available in developed countries, these techniques have the ability to identify cancer at the very initial stages.

\section{Case Study}

Male breast cancer is already a very rare type of cancer, moving onto its subtypes micropapillary cancer is a rarer subtype of invasive ductal carcinoma. Micropapillary carcinoma is an extremely scanty subtype and very rarely malignant. Micropapillary carcinoma is often associated to axillary lymph node metastasis. Micropapillary carcinomas are assessed over the presence of papillary breast lesions. histopathological analysis plays a key role in the accurate diagnosis of micropapillary carcinoma.

\section{Background}

Herein we discuss a case reported in a local hospital of Pakistan. This was a rare case which reported recently. A 50-yearold man reported the presence of a lump in his breast. After further examinations the therapies were recommended. The patient had a hard lump in the breast for almost three years when the subject was brought under consideration following test were run for the discernment of the lump that either it be cancerous or not.

\section{Testing}

Some tests were run to confirm that either the lump is cancerous or not.

Cytology Test: It confirmed the suspiciousness of the lump. At that time the lump was hard was about the size of $6 \times 5 \mathrm{~cm}$ hard. It was diagnosing to be a class three smear which is highly suspicious. The cytological reports confirmed it to be unusual, but it was most likely to begin.

Biopsy: For the further evaluation of the lump it Is further sent for biopsy which confirmed the presence of papillary lesions composed of fibrovascular cores. The sample showed negative results to the following immunohistochemical stains. 
1. $\mathrm{P} 63$

2. $\mathrm{Ck} 5 / 6$

3. Myosin

All these results diagnosed it to be papillary neoplasm, complete excision of the lump was recommended at that time.

Bone Scan \& Ct Cap: Bone scan showed normal results and CT cap confirmed papillary neoplasm of right breast with pulmonary soft tissue nodules.

Stage and Type: The above-mentioned test certified the presence of a cancerous lump. The stage of cancer was labelled as T1NoMo with a breast lump of $4.2 \times 7 \mathrm{~cm}$ with both solid and cystic compounds.

\section{Treatment}

The patient went into MRM of the right breast. After that he went into radiotherapy. The patient secured 2 cycles of SSD therapy with $100 \mathrm{~cm}$ in both fields on a daily dose of 220 . After that the patient went for further evaluation which is unknown to us.

\section{Discussion}

The reason for discussing this case is to discuss and compare the therapeutic follow-up that is commonly adopted in Pakistan for the management of male breast cancer. Therefore, the methods that are opted for the diagnosis and management of male breast cancer are ideal $[33,34]$. As per the international standards the first and foremost method of identification is physical examination which is followed by clinical testing, both these methods are well employed and well utilized Furthermore, the treatment strategies opted was also according to the marked standards. Lumpectomy was then followed by SSD radiation cycles after that the patient was reassessed for the recurrence of any kind of cancerous mass Despite of all these factors Pakistan is still unable to provide novel identification techniques so that's why breast cancer in both males and females is not identified at initial stages Moreover, Pakistan lacks established treatment protocols and genetic testing, even if these settlements are available they are not as widespread as they should be. Therefore, the survival rate is a bit lower in Pakistan just because of these few reasons $[35,36]$.

Another factor that is the most prevalent cause for the poor prognosis of such patients is the financial limitation, Pakistan is a developing country therefore it is very difficult for people to afford such costly treatments and medication. Commonly employed medicine for male breast cancer trastuzumab is mostly cut down by the doctors because it's out of the financial range of the patients The main reason to discuss such unusual cases is that Pakistan should establish its own databases that cover all the male breast cancer cases occurring in the country also it should be widely accessible for everyone so it might raise awareness among people that breast cancer is not only confined to the female body but it can also spread to males as well $[37,38]$.

\section{Future Prospective}

The future perspectives for the treatment of male breast cancer or breast cancer in general are a novel therapy that is characterized as microRNA therapy. MicroRNA has been widely studied for its role in the identification of several types of cancer but its unique property of gene silencing is overall.

\section{Microrna Therapy}

The microRNA therapy can be applied but not enough studies are there to support it. secondly there are a number of barriers that hinder the use of miRNA therapy, the barriers are listed below

1. There is not enough evidence of clinical trials

2. Production of a synthetic miRNA that can adapt with the human body

3. For the utilization of miRNA, toxicity and antigenicity should be brought to consideration

4. There is no appropriate delivery system for the sitespecific delivery of miRNA

Removing these barriers, we can bring miRNA therapy to light, but this requires a little bit of more research in the concerned area (94).

\section{Nanoparticles}

Nanoparticles are a new and completely novel concept. It has attracted researchers' attention because of their robustness. Nanoparticles exhibit versatile characteristics they have multiple uses and can be employed for different medicinal purposes. There are several uses for the application of nanoparticles

1. They are excellent vectors for drug delivery.

2. They are used for the identification of cancer at initial stages

3. The discovery of nanobots has opened new dimensions in the field of therapeutics. Nanobots can repair the defective cells at the site of the disease with full efficiency

4. Nanoparticles are safer vectors because of their antimicrobial properties

Nano particles are widely employed in the therapies of breast cancer because of the adverse effects of the therapeutics used in the treatment of breast cancer. New techniques have been devised by employing the nano particles to coat the drugs that are used for the therapies of the breast cancer.

Small Interfering RNA (SIRNA) are mounted on the nano carriers for targeted therapeutic delivery SIRNA is already a widely studied topic because of their marvelous character of gene silencing. Glucose mounted AuNPs, with SIRNA delivered on the site of the tumor is used because glucose is overused by cancerous cells because of their high metabolic activity (95). Furthermore, 
research is needed in the area because of some limitations. But all the techniques discussed above are promising designs but need furthermore research to be approved by FDA and for error free manipulation.

\section{References}

1. Giordano SH (2005) A review of the diagnosis and management of male breast cancer. Oncologist 10(7): 471-479.

2. Bray F, Ferlay J, Soerjomataram I, Siegel RL, Torre LA, et al. (2018) Global cancer statistics 2018: GLOBOCAN estimates of incidence and mortality worldwide for 36 cancers in 185 countries. CA Cancer J Clin 68(6): 394424.

3. Ottini L (2014) Male breast cancer: A rare disease that might uncover underlying pathways of breast cancer. Nat Rev Cancer 14(10): 643.

4. Yalaza M, Inan A, Bozer M (2016) Male breast cancer. J Breast Health 12(1): 1-8.

5. Brinton LA, Richesson DA, Gierach GL, Lacey JV Jr, Park Y, et al. (2008) Prospective evaluation of risk factors for male breast cancer. Journal Natl Cancer Inst 100(20): 1477-1481.

6. Jemal A, Thomas A, Murray T, Thun M (2002) Cancer statistics, 2002. Ca Cancer J Clin 52(1): 23-47.

7. Khokher S, Qureshi MU, Riaz M, Akhtar N, Saleem A (2012) Clinicopathologic profile of breast cancer patients in Pakistan: Ten years data of a local cancer hospital. Asian Pac J Cancer Prev 13(2): 693-698.

8. Fentiman IS, Fourquet A, Hortobagyi GN (2006) Male breast cancer Lancet 367(9510): 595-604.

9. Weiss JR, Moysich KB, Swede H (2005) Epidemiology of male breast cancer. Cancer Epidemiol Biomarkers Prev 14(1): 20-26.

10. Korde LA, Zujewski JA, Kamin L, Giordano S, Domchek S, et al. (2010) Multidisciplinary meeting on male breast cancer: Summary and research recommendations. J Clin Oncol 28(12): 2114-2122.

11. Atalay C, Kanlioz M, Altinok M (2003) Prognostic factors affecting survival in male breast cancer. J Exp Clin Cancer Res 22(1) :29-33.

12. Koc M, Polat P (2001) Epidemiology and aetiological factors of male breast cancer: A ten years retrospective study in eastern Turkey. Eur J Cancer Prev 10(6): 531-534

13. Rizzolo P, Silvestri V, Tommasi S, Pinto R, Danza K, et al. (2013) Male breast cancer: Genetics, epigenetics, and ethical aspects. Ann Oncol 24: viii75-viii82.

14. Giordano SH, Buzdar AU, Hortobagyi GN (2002) Breast cancer in men. Ann Intern Med 137: 678-687.

15. Martin AM, Weber BL (2000) Genetic and hormonal risk factors in breast cancer. J Natl Cancer Inst 92(14): 1126-1135.

16. Wasielewski M, den Bakker MA, van den Ouweland A, Meijer-van Gelder ME, Portengen H, et al. (2009) CHEK2 1100delC and male breast cancer in the Netherlands. Breast Cancer Res Treat 116(2): 397-400.

17. Thellenberg C, Malmer B, Tavelin B, Grönberg H (2003) Second primary cancers in men with prostate cancer: An increased risk of male breast cancer. J Urol 169(4): 1345-1348.

18. Brinton LA, Carreon JD, Gierach GL, McGlynn KA, Gridley G (2010) Etiologic factors for male breast cancer in the US Veterans Affairs medical care system database. Breast Cancer Res Treat 119(1): 185-192.

19. Giordano SH (2018) Breast cancer in men. N Engl J Med 378(24): 23112320.
20. Fostira F, Saloustros E, Apostolou P, Vagena A, Kalfakakou D, et al. (2018) Germline deleterious mutations in genes other than BRCA2 are infrequent in male breast cancer. Breast Cancer Res Treat 169(1): 105113.

21. Hill TD, Khamis HJ, Tyczynski JE, Berkel HJ (2005) Comparison of male and female breast cancer incidence trends, tumor characteristics, and survival. Ann Epidemiol 15(10): 773-780.

22. Giordano SH, Cohen DS, Buzdar AU, Perkins G, Hortobagyi GN (2004) Breast carcinoma in men: A population-based study. Cancer 101(1): 5157.

23. Fentiman IS (2016) Male breast cancer is not congruent with the female disease. Crit Rev Oncol Hematol 101: 119-124.

24. Losurdo A, Rota S, Gullo G, Masci G, Torrisi R (2017) Controversies in clinicopathological characteristics and treatment strategies of male breast cancer: A review of the literature. Crit Rev Oncol Hematol 113: 283-291.

25. Javidiparsijani S, Rosen LE, Gattuso P (2017) Male breast carcinoma: A clinical and pathological review. Int J Surg Pathol 25(3): 200-205.

26. Pritzlaff M, Summerour P, McFarland R, Li S, Reineke P, et al. (2017) Male breast cancer in a multi-gene panel testing cohort: Insights and unexpected results. Breast Cancer Res Treat 161(3): 575-586.

27. Kiluk JV, Lee MC, Park CK, Meade T, Minton S, et al. (2011) Male Breast Cancer: Management and Follow-up Recommendations. Breast J 17(5): 503-509.

28. Easton DF, Pharoah PD, Antoniou AC, Tischkowitz M, Tavtigian SV, et al. (2015) Gene-panel sequencing and the prediction of breast-cancer risk. N Engl J Med 372: 2243-2257.

29. Hauke J1, Horvath J2, Groß E3, Gehrig A4, Honisch E, et al. (2018) Gene panel testing of 5589 BRCA1/2-negative index patients with breast cancer in a routine diagnostic setting: Results of the German Consortium for Hereditary Breast and Ovarian Cancer. Cancer Med 7(4): 1349-1358.

30. (2017) American Cancer Society. Cancer-facts-and-figures.

31. Evans GF, Anthony T, Turnage RH, Schumpert TD, Levy KR, et al. (2001) The diagnostic accuracy of mammography in the evaluation of male breast disease. Am J Surg 181(2): 96-100.

32. Günhan-Bilgen I, Bozkaya H, Ustün E, Memiş A (2002) Male breast disease: Clinical, mammographic, and ultrasonographic features. Eur J Radiol 43(3): 246-255.

33. Giordano SH, Valero V, Buzdar AU, Hortobagyi GN (2002) Efficacy of anastrozole in male breast cancer. Am J Clin Oncol 25(3): 235-237.

34. Zabolotny BP, Zalai CV, Meterissian SH (2005) Successful use of letrozole in male breast cancer: A case report and review of hormonal therapy for male breast cancer. J Surg Oncol 90(1): 26-30.

35. Siegel R, Ma J, Zou Z, Jemal A (2014) Cancer statistics. CA cancer j clin 64(1): 9-29.

36. Rizzolo P, Zelli V, Silvestri V, Valentini V, Zanna I, et al. (2019) Insight into genetic susceptibility to male breast cancer by multigene panel testing: Results from a multicenter study in Italy. Int J Cancer 145(2): 390-400.

37. Vimalraj S, Miranda P, Ramyakrishna B, Selvamurugan N (2013) Regulation of breast cancer and bone metastasis by microRNAs. Dis mark 35(5): 369-387.

38. Yi Y, Kim HJ, Zheng M, Mi P, Naito M, et al. (2019) Glucose-linked sub-50nm unimer polyion complex-assembled gold nanoparticles for targeted siRNA delivery to glucose transporter 1-overexpressing breast cancer stem-like cells. J Control Release 295: 268-277. 
ISSN: 2574-1241

DOI: $10.26717 /$ BJSTR.2019.21.003645

Sadia Zia. Biomed J Sci \& Tech Res

(C) 7 This work is licensed under Creative

Submission Link: https://biomedres.us/submit-manuscript.php

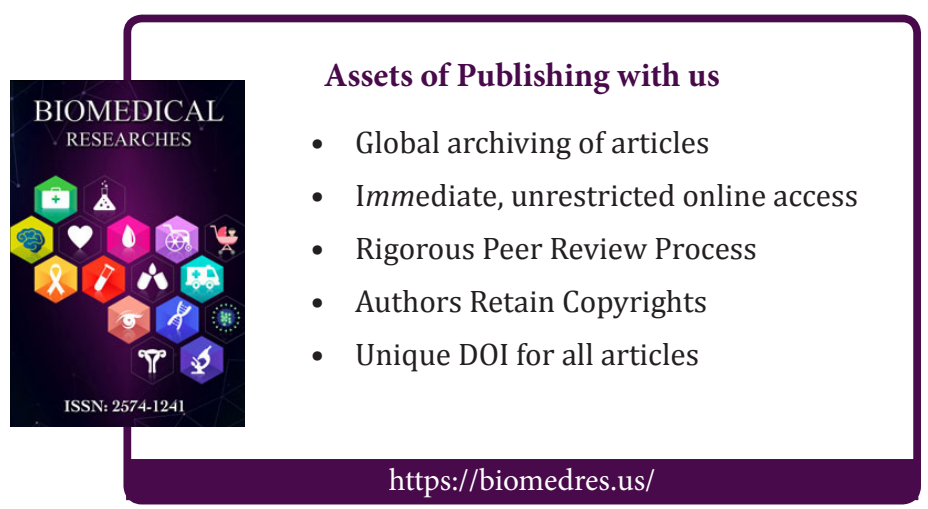

